\title{
A influência dos ativos intangíveis na criação de valor de empresas norte-americanas do setor de serviços ao consumidor
}

Erica Sumoyama Braune ${ }^{1}$, Juliana Albuquerquer Saliba ${ }^{1}$, Leonardo Fernando Cruz Basso ${ }^{1}$, Herbert Kimura ${ }^{1}$

\footnotetext{
${ }^{1}$ Universidade Presbiteriana Mackenzie.

Correspondência: leonardobasso@mackenzie.br
}

Resumo As empresas estão focando nos ativos intangíveis com a intenção de obter vantagem competitiva, no entanto, não há uma forma sistemática de calculá-los. Gu e Lev (2003) propõem um modelo de mensuração dos ativos intangíveis, onde o desempenho econômico da empresa é gerado por ativos físicos, financeiros e intangíveis. Baseado nessa proposta, calculamos o intangible capital $(\mathrm{IC})$, o intangiblesdrive-earnings (IDE) e os índices de desempenho para empresas do setor de serviços ao consumidor dos Estados Unidos de acordo com a disponibilidade de dados no período de 1998 a 2010. Utilizamos o modelo de dados em painel, para analisarmos simultaneamente as empresas e suas variáveis ao longo do tempo. Os resultados mostram que o comprehensive value tem uma relação positiva e significante com o Valor de Mercado e que o IC e do IDE possuem uma relação positiva e significante com as variáveis de pesquisa e desenvolvimento e dispêndio de capital, variáveis de demonstrações financeiras que são comumente usadas como proxies para os intangíveis. A pesquisa pode ser ampliada para empresas publicas onde seja possível a obtenção de variáveis oriundas de balanços e para as quais seja possível calcular o valor de mercado. Empresas públicas não podem mais ser tratadas como entidades diferenciadas de empresas privadas no que concerne a avaliação de desempenho, pois a ineficiência pública vai implicar perda de eficiência para as empresas privadas. Assim modelos como 
os que testamos aqui poderiam ser testados no setor público para verificar se resultados semelhantes são obtidos.

Palavras-chave: ativos intangíveis, criação de valor, retorno total ao acionista.

Abstract

The firms are focusing in intangible assets intending to obtain competitive advantage; nevertheless, there is no standardized manner to calculate such assets. Gu and Lev (2003) proposed a model for measuring intangible assets, considering that the firms' economic performance is a result of tangible, financial and intangible assets. Based on this proposal, we calculated the intangible capital (IC), intangibles-drive-earnings (IDE) and performance indexes for firms on U.S. consumer services sector, according to availability of data during the period from 1998 to 2010. Panel data modeling was used to analyze simultaneously firms and its variables along time. Results demonstrate that the comprehensive value presents a positive and significant relation with market value and that the IC and the IDE have positive and significant relation with research and development variables and capital variables; variables from financial demonstrations that are usually employed as proxies for intangible assets. The research may be extended for public enterprises with information on balance sheets and market value. Public enterprises should not be considered different from private firms in performance evaluation, since public sector inefficiency may generate private losses in efficiency. Thus, similar models to the one presented in this paper may be tested for public sector evaluation, in order to compare results obtained.

Keywords: intangible assets, value creation, shareholder total profit.

Resumen Las empresas tienen foco en los activos intangibles para obtener ventajas competitivas; pero no hay una forma estandarizada de calcular-se. Gu y Lev (2003) proponen un modelo para mensuración de los activos intangibles, que considera el performance económico de la empresa un resultado generado por los activos físicos, financieros y intangibles. Basado en esa propuesta, calculamos el intangible capital (IC), el intangibles-drive-earnings (IDE) y los índices de performance para empresas del sector de servicios al consumidor de los Estados Unidos con informaciones del período de 1998 hasta 2010. Utilizamos el modelo de dados en painel para analizar simultáneamente las empresas y sus variables envolviendo en el tempo. Los resultados muestran que el comprehensive value tiene una relación positiva y significante con el Valor de Mercado y que el IC y el IDE presentan una relación positiva y significante con las variables de pesquisa y desenvolvimiento y gasto de capital, variables de demonstraciones financieras comúnmente usadas como proxies para los intangibles. La pesquisa puede ser ampliada para empresas públicas con informaciones contables y valor de mercado. Empresas públicas no pueden ser tratadas como entidades diferenciadas de las empresas privadas en la evaluación de performance, porque la ineficiencia pública implica reducción de la eficiencia de las empresas privadas. Así, modelos como el presentado pueden ser testados en el sector público para verificar si los resultados equipárense.

Palabras-clave: activos intangibles, creación de valor, retorno total al accionista. 


\section{Introdução}

Para Lev (2001), a economia atual é movida principalmente por ativos intangíveis. Um dos motivos é porque as empresas estão dependendo cada vez mais da inovação para sua sobrevivência e crescimento devido à intensificação da concorrência empresarial e as facilidades geradas pela tecnologia da informação.

Villalonga (2004), baseada na teoria da RBV realizou um estudo que verificou que quanto maior o grau de intangibilidade dos recursos da empresa, maior é sua vantagem competitiva sustentável, sendo assim, este também é um motivo para as empresas focarem nos ativos intangíveis, para que consigam se diferenciar no mercado.

Além da importância dos ativos intangíveis para as empresas serem mais competitivas, Lev (2001) identificou que os principais grupos de interesse nos ativos intangíveis são:

(i) Administradores de empresas e acionistas: os investimentos em intangíveis estão associados aos custos excessivos de capitais, sendo que os administradores e acionistas estão interessados em diminuir os custos adicionais de capital;

(ii) Investidores e reguladores do mercado de capitais: a assimetria das informações pode gerar conseqüências indesejáveis e por isso, os investidores estão interessados em reduzir a informação assimétrica causada pelos intangíveis;

(iii) Contadores e conselho de administração: a contabilização deficiente dos intangíveis facilita os vieses e os relatórios financeiros fraudulentos e com isso, os conselhos de administração dependem das informações contábeis para monitorar as atividades gerenciais;

(iv) Classe política: as deficiências das informações dos ativos intangíveis afetam as políticas públicas, assim, as demonstrações financeiras das empresas são importantes para as contas nacionais e deliberações políticas.

Apesar do grande interesse em se mensurar os intangíveis, Low e Kalafut (2002) verificaram que os especialistas se baseiam nos ativos intangíveis para analisar o potencial e o desempenho das empresas, mas que esta análise ainda não é feita de forma sistemática, pois a maioria das empresas não consegue demonstrar aos seus investidores suas métricas e como são calculadas.

Low e Kalafut (2002) também analisaram que as empresas de serviços são diferentes das indústrias, pois embora precisem de instalações, não exigem tantos ativos tangíveis como uma fábrica, ou seja, a maior parte de seu valor depende de profissionais altamente qualificados para prestar o serviço desejado aos seus clientes. De acordo com esse argumento, pretendemos estudar neste 
artigo empresas do setor de serviços, pois possuem uma dependência grande dos ativos intangíveis.

O presente artigo pretende verificar a partir de dados em painel entre os anos de 1998 e 2010, o modelo proposto por Gu e Lev (2003) para mensuração dos ativos intangíveis de empresas de serviços ao consumidor dos Estados Unidos, baseado no IDE (intangibles-driven-earnings) e no IC (intangible capital), para analisar a sua influência na criação de valor das empresas através dos índices de intangibilidade e a relação com as variáveis de pesquisa e desenvolvimento, dispêndio de capital e despesas administrativas. Nesse contexto, pretendemos responder ao seguinte problema de pesquisa: Qual a influência dos ativos intangíveis na criação de valor das empresas de serviços ao consumidor?

Este artigo está estruturado da seguinte forma: no próximo tópico apresentaremos o referencial teórico, em seguida descreveremos a metodologia de pesquisa, a análise dos resultados e no último tópico, as considerações finais.

\section{Referencial teórico}

\section{Ativos intangíveis}

Os ativos intangíveis são aqueles que não possuem existência física, mas representam valor para a empresa, eles são tipicamente de longo prazo e de difícil avaliação (Edvinsson e Malone 1997). Segundo Lev (2001:5), um ativo intangível é "um direito a benefícios futuros que não possui uma forma física ou financeira". Para Daum (2005), os ativos intangíveis são recursos não materiais que desempenham um papel fundamental no processo de criação de valor de uma empresa que lhe permitem competir com êxito.

Para Stewart (1997), o ativo intangível é constituído pelo talento dos funcionários, a eficácia dos sistemas gerenciais e do caráter de seus relacionamentos com os clientes e contribuem mais para o valor final de um produto do que os ativos tangíveis.

Segundo Domeneghetti e Meir (2009), uma fonte de ativo intangível é a tecnologia da informação, que vem se transformando e se tornando mais complexa, pois está envolvida com planejamento e metas além de ser uma extensão das habilidades dos funcionários.

Para Low e Kalafut (2002), o valor dos intangíveis está na habilidade e no conhecimento dos administradores da empresa, ou seja, que depende das relações e da reputação que a empresa estabelece com seus fornecedores, cliente, parceiros e acionistas e da forme como ela opera. Para eles os principais elementos dos intangíveis são: liderança, estratégia, comunicações, marca, 
reputação, alianças e redes, tecnologia, capital humano, local de trabalho, cultura, inovação e capacidade de adaptação.

Analisando alguns autores podemos encontrar diversos elementos que podem constituir os ativos intangíveis. Baseado nisso, Sullivan (2000) fez uma coletânea dos componentes dos ativos intangíveis como mostra a Figura 1.

Segundo Sullivan (2000), a diversidade de opinião sobre quais são os elementos dos ativos intangíveis é resultado de uma ampla gama de interesses e perspectivas, sendo que cada definição é compatível com as perspectivas e interesses de cada usuário que negligencia ou ignora os interesses e perspectivas dos outros.

Figura 1. Elementos dos ativos intangíveis.

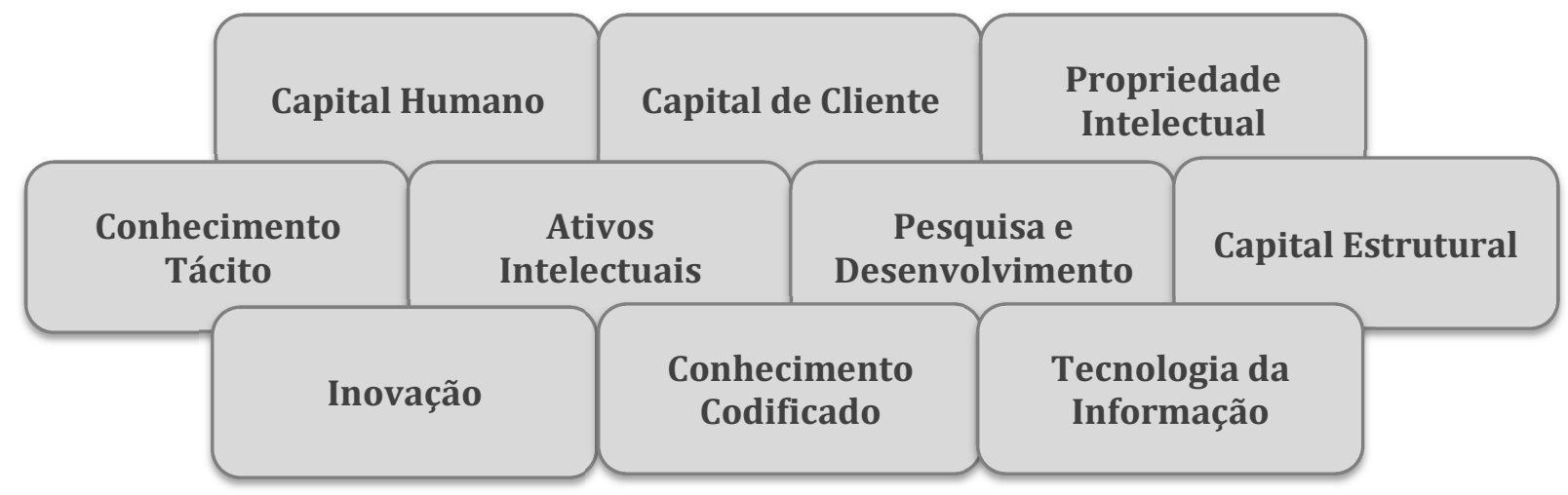

Fonte: adaptado de Sullivan (2000).

\section{Modelo de mensuração de Gu e Lev (2003)}

Gu e Lev (2003) propõem um método diferente para estimar o valor dos ativos intangíveis baseado no conceito econômico de "função de produção", onde o desempenho econômico da empresa será gerado por ativos físicos, financeiros e intangíveis, a partir da seguinte fórmula:

Equação 1

$$
D E=\alpha A F+\beta A F I N+\gamma A I
$$

Onde $\mathrm{DE}=$ desempenho econômico, $\mathrm{AF}=$ ativos físicos, $\mathrm{AFIN}=$ ativos financeiros, $\mathrm{Al}=$ ativos intangíveis e $\alpha, \beta$ e $\gamma$ representam as contribuições dos ativos. 
Este método será utilizado neste artigo para verificamos a influência dos ativos intangíveis na criação de valor das empresas, pois diferente dos outros métodos, Gu e Lev (2003) propõe calcularmos o estoque de ativos intangíveis e não o fluxo dos intangíveis.

Gu e Lev (2003) denominam como intangibles-driven-earnings (IDE) a manipulação da equação do desempenho econômico conforme demonstrado abaixo:

Equação 2

$$
I D E=D E-\alpha A F+\beta A F I N
$$

Com base nessa equação, Gu e Lev (2003) propõe cinco etapas para projeção do IDE e cálculo do estoque de ativos intangíveis.

Figura 2. Resumo das etapas do método de Gu e Lev (2003).

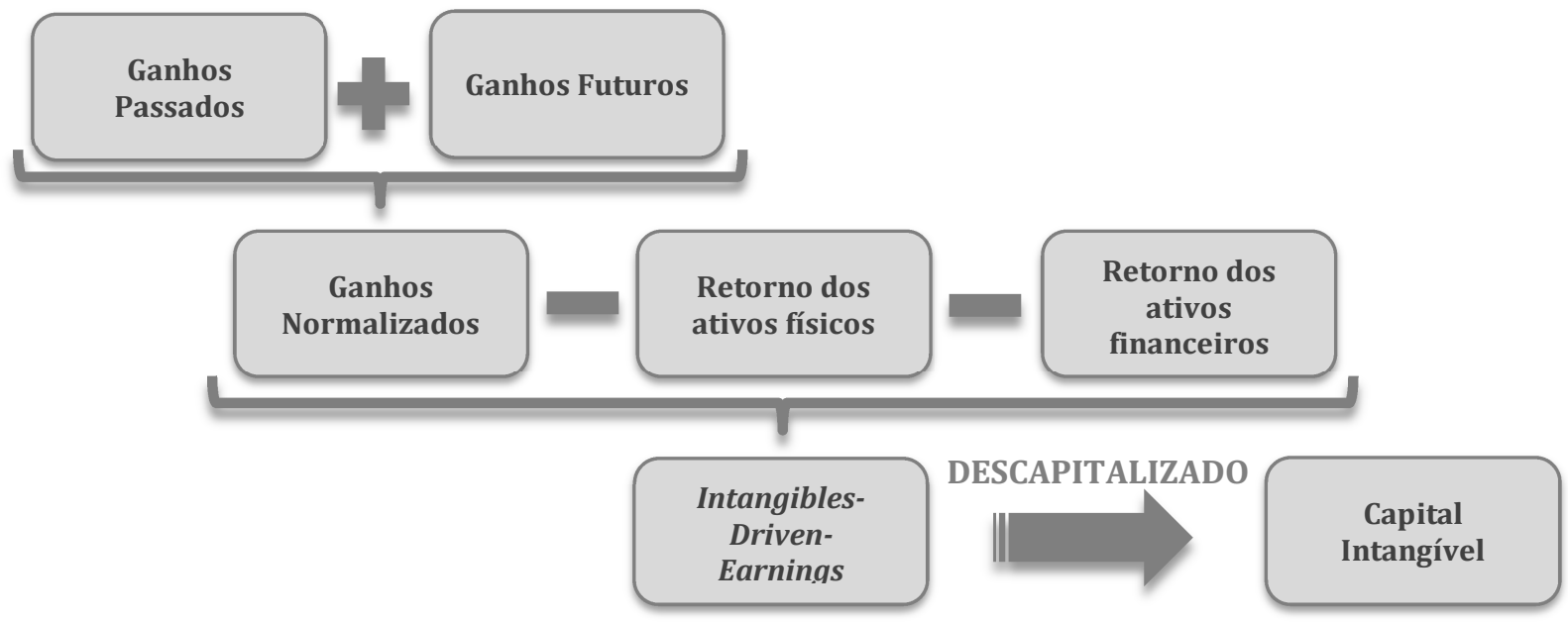

Fonte: adaptado de Gu e Lev (2003).

\section{Etapa 1 - Cálculo do desempenho econômico}

Para o cálculo do desempenho econômico, Gu e Lev (2003) sugerem a utilização de três a cinco anos e, quanto maior o ano, maior deve ser a ponderação.

Neste artigo, calculamos o desempenho econômico (DE) com base nos resultados de quatro anos passados e nas projeções de quatro anos futuros do EBITDA. Contudo, como o objetivo deste trabalho não é verificar o poder preditivo do 
modelo, utilizamos dados realizados também para os anos futuros, mas dessa forma restringimos o cálculo do desempenho econômico até o ano de 2006, considerando que temos dados até o ano de 2010, como mostra a Figura 3.

Figura 3. Período de cálculo do desempenho econômico.

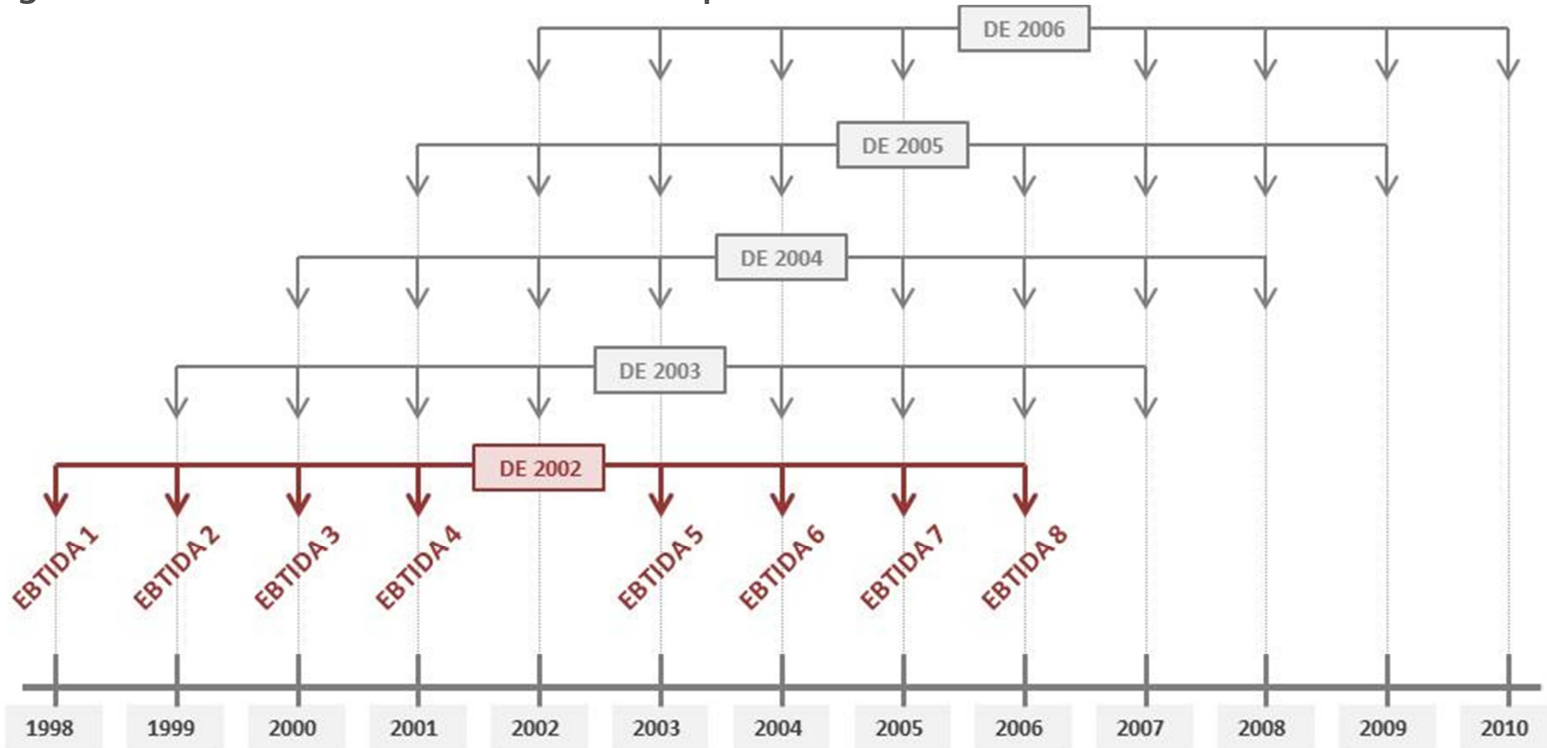

Fonte: elaboração dos autores.

Como Gu e Lev não deixam explícitas as ponderações, utilizaremos a seguinte equação:

Equação 3

$$
x_{n}=n \times\left(\frac{1}{\sum_{n=1}^{8} n}\right)
$$

Assim, temos que $x_{n}=n \times 2,7778 \%$ e a seguinte equação do desempenho econômico:

\section{Equação 4}

$$
D E=\sum_{n=1}^{8} x_{n} \times E B I T D A_{n}
$$


Ao fim desta etapa temos o valor do desempenho econômico dos anos de 2002, 2003, 2004, 2005 e 2006.

\section{Etapa 2 - Cálculo dos ativos físicos e financeiros}

Segundo Gu e Lev (2003), os ativos físicos são as propriedades, plantas e equipamentos e os ativos financeiros são as ações, o dinheiro em caixa e os instrumentos financeiros.

Baseado em estimativas apresentadas em estudos anteriores em economia e finanças, Gu e Lev (2003) propõe a utilização da taxa de 7\% após impostos, para os ativos físicos $(\alpha)$ e $4,5 \%$ para os ativos financeiros $(\beta)$, refletindo médias para toda a economia americana.

\section{Etapa 3 - Estimação do IDE}

Nesta etapa, substituímos os valores encontrados nas etapas 1 e 2 na equação do IDE para estimarmos o valor de cada ano.

Assim, para calcularmos o IDE utilizamos a seguinte equação:

Equação 5

$$
I D E=D E-7,0 \% \times A F-4,5 \% \times A F I N
$$

\section{Etapa 4 - Cálculo do IDE para três períodos futuros}

Na etapa 4 prevemos a série de IDEs em três períodos futuros com base em um modelo de avaliação em três fases.

No primeiro período, de 1 a 5 anos, utilizamos os IDEs calculados na etapa anterior. No segundo período, de 6 a 10 anos, convergimos linearmente os IDEs até chegar a uma taxa de crescimento de 3\% e no terceiro período, de 11 anos até o infinito, utilizamos uma taxa de crescimento de $3 \%$ ao ano, conforme proposto por Gu e Lev (2003).

A Figura 4 demonstra a série de IDEs dos três períodos. 
Figura 4. Série de IDEs dos três períodos.

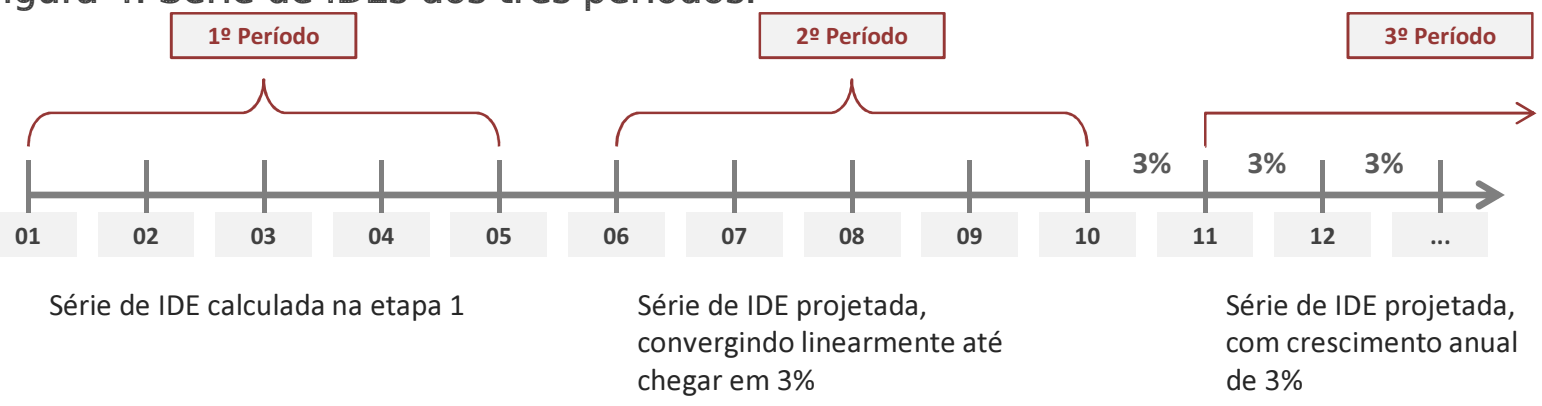

Fonte: elaboração dos autores.

\section{Etapa 5 - Determinação do estoque de capital intangível}

Nesta etapa calcularemos a série de IDEs descontados a uma taxa que reflita o grau de risco para determinarmos o estoque de capital intangível.

Segundo Daum (2001), riscos associados ao investimento em bens intangíveis, especialmente dos investimentos na estratégia e na cadeia de inovação de produto de uma empresa, são muito maiores do que no tipo de ativos tradicionais.

Baseado nisso, utilizaremos uma taxa de $7,5 \%$, pois os ativos intangíveis devem refletir uma taxa maior que os ativos financeiros. Assim temos a seguinte equação:

\section{Equação 5}

$$
I C_{n}=\sum_{k=n}^{\infty} I D E_{k} /(1+i)^{k-n}
$$

Onde IC = intangible capital, $i$ é a taxa dos ativos intangíveis, $k$ o ano do IDE e $n$ o ano do IC.

Gu e Lev (2003) propõem alguns índices de avaliação de desempenho utilizando o intangibles-driven-earnings (IDE) ou o intangible capital (IC), com a intenção de avaliar a relevância dos intangíveis quando comparados a medidas convencionais de desempenho, como o lucro. São eles: 
Tabela 1. Série de IDEs dos três períodos.

\begin{tabular}{lc}
\hline Variável & Definição \\
\hline Margem do capital intangível (ICM) & $I C M=\frac{I C}{\text { Vendas }}$ \\
\hline Margem de ganhos intangíveis (IDEM) & $I D E M=\frac{I D E}{\text { Vendas }}$ \\
\hline Margem do capital intangível operacional (ICOM) & ICOM $=\frac{I D E}{\text { Lucro Operacional }}$ \\
\hline Razão entre o capital intangível e o valor contábil (ICR) & ICR $=\frac{I C}{\text { Valor Contábil }}$ \\
\hline $\begin{array}{l}\text { Comprehensive value (CV) } \\
\text { Razão entre o valor de mercado e o comprehensive value } \\
\text { (MVR) }\end{array}$ & MV R $=\frac{\text { Valor de Mercado }}{C V}$ \\
\hline
\end{tabular}

Fonte: adaptado de Gu e Lev (2003).

Como um dos objetivos do estudo é identificar o impacto dos intangíveis na criação de valor, é importante discutir quais medidas de desempenho são relevantes. Para Young e O'Byrne (2003), geralmente os altos executivos das empresas são pagos para responder coisas diferentes da criação de valor e sendo assim a criação de valor acaba não sendo prioridade para os administradores. Isso causa uma destruição de valor, pois os objetivos dos administradores podem ser conflitantes com a criação de valor. No entanto as empresas precisam criar valor, pois são imprescindíveis para garantir a geração de riqueza para os acionistas em longo prazo. Com base nisso, as empresas precisam adotar medidas de desempenho para controlar e acompanhar a criação de valor, para que os funcionários trabalhem de acordo com os objetivos dos acionistas. Neste artigo usamos retorno total ao acionista (TSR) como uma variável de criação de valor, como proposto por Gu e Lev (2003).

\section{Metodologia}

A característica desta pesquisa é descritiva e tem como objetivo investigar os índices e variáveis de intangibilidade, conforme modelo explicado no tópico anterior, sua relação com o valor de mercado das empresas e com o retorno ao acionista. Baseado nisso e com o propósito de respondermos ao problema de pesquisa proposto neste artigo, formulamos as seguintes hipóteses e suas respectivas equações: 
Hipótese 1: O valor de mercado (MV) é positivamente relacionado com o comprehensive value (CV).

$$
H_{0,1}: M V_{i t}=\beta_{0}+\beta_{1} C V_{i t}+v_{i t}
$$

Hipótese 2: Quanto maior o intangible capital (IC) das empresas, maior é o investimento em pesquisa e desenvolvimento (PD), o dispêndio de capital (CAPEX) e as despesas administrativas (SGA).

$$
H_{0,2}: I C_{i t}=\beta_{0}+\beta_{1} P D_{i t}+\beta_{2} C A P E X_{i t}+\beta_{3} S G A_{i t}+v_{i t}
$$

Hipótese 3: Quanto maior o intangibles-driven-earnings (IDE) das empresas, maior é o investimento em pesquisa e desenvolvimento (PD), o dispêndio de capital (CAPEX) e as despesas administrativas (SGA).

$$
H_{0,3}: I D E_{i t}=\beta_{0}+\beta_{1} P D_{i t}+\beta_{2} C A P E X_{i t}+\beta_{3} S G A_{i t}+v_{i t}
$$

Hipótese 4: Quanto maior o retorno ao acionista (TSR), maior é o IDE das empresas considerando os ganhos (EARN = EBITDA) como variável de controle.

$$
H_{0,4}: T S R_{i t}=\beta_{0}+\beta_{1} I D E_{i t}+\beta_{2} \Delta I D E_{i t}+\beta_{3} E A R N_{i t}+\beta_{4} \Delta E A R N_{i t}+v_{i t}
$$

Hipótese 5: Quanto maior a margem de capital intangível (ICM), maior a criação de valor.

$$
H_{0,5}: T S R_{i t}=\beta_{0}+\beta_{1} I M C_{i t}+v_{i t}
$$

Hipótese 6: Quanto maior a margem de ganhos intangíveis (IDEM), maior a criação de valor.

$$
H_{0,6}: T S R_{i t}=\beta_{0}+\beta_{1} I D E M_{i t}+v_{i t}
$$

Hipótese 7: Quanto maior a margem de capital intangível operacional (ICOM), maior a criação de valor.

$$
H_{0,7}: T S R_{i t}=\beta_{0}+\beta_{1} I C O M_{i t}+v_{i t}
$$

Hipótese 8: Quanto maior a razão entre o capital intangível e o valor contábil (ICR), maior a criação de valor.

$$
H_{0,8}: T S R_{i t}=\beta_{0}+\beta_{1} I C R_{i t}+v_{i t}
$$


Hipótese 9: Quanto maior a razão entre o valor de mercado e o comprehensive value (MVR), maior a criação de valor.

$$
H_{0,9}: T S R_{i t}=\beta_{0}+\beta_{1} M V R_{i t}+v_{i t}
$$

Foram utilizados dados secundários do banco de dados Thomson-Reuters levantados no Datastream. Selecionamos para a amostra empresas dos Estados Unidos de acordo com sua disponibilidade de dados no período de 1998 a 2010 do setor de serviços ao consumidor com um total de 206 empresas. A escolha do setor baseou-se em sua grande dependência dos ativos intangíveis de acordo com o argumento de Low e Kalafut (2002) de que as empresas de serviços dependem mais dos ativos intangíveis do que as indústrias.

Neste artigo, utilizamos o modelo de dados em painel, pois podemos combinar séries temporais e dados de corte transversal, ou seja, nos permite analisar simultaneamente as empresas e suas variáveis ao longo do tempo. Para a análise foram consideradas apenas as variáveis no período de 2002 a 2006, ou seja, para um período de cinco anos, pois os períodos de 1998 à 2001 e 2007 à 2010 foram utilizados para o cálculo do IDE, conforme modelo de Gu e Lev (2003) detalhado no tópico anterior.

\section{Análise dos resultados}

Para cada hipótese, mostramos as estatísticas descritivas da amostra utilizada, os resultados do modelo de dados em painel e os resultados da regressão. A maioria dos modelos possui heterocedasticidade e alguns modelos possuem autocorrelação. A fim de reduzir a influência de características indesejáveis dos dados, utilizamos erros robustos para modelos de efeitos fixos e mínimos quadrados generalizados para modelos de efeitos aleatórios.

Para determinar qual modelo de dados do painel é o mais apropriado, nós executamos três testes estatísticos: o F-teste, para verificar a melhor opção entre o modelo de coeficientes constantes (polled) e o modelo de efeitos fixos; o teste Breush-Pagan para verificar a melhor opção entre o modelo de coeficientes constantes e do modelo de efeitos aleatórios; e o teste de Hausman para verificar a melhor opção entre o modelo de efeitos fixos e o modelo de efeitos aleatórios. Os resultados das regressões são apresentados nas Tabelas 1 e 2 .

Resultados da análise de dados em painel sugerem que o comprehensive value é positivamente relacionada com o valor de mercado das empresas no setor de serviços ao consumidor dos Estados Unidos, corroborando Hipótese 1. Este resultado sugere que os investidores podem atribuir um valor intangível que pode ser refletida no constructo do capital intangível IC desenvolvido por Gu e Lev (2003). Em relação as variáveis de demonstrações financeiras para denotar 
os intangíveis, estudo da Hipótese 2 mostra que o investimento em pesquisa e desenvolvimento (RD), bem como as despesas de capital (CAPEX) são significativamente e positivamente relacionadas com o constructo IC. Vendas, gerais e administrativas não são significativamente relacionados à $I C$. Devido à sua metodologia, o IC pode ser considerado uma medida inovadora da intangibilidade, pois é significativamente relacionado com variáveis que são frequentemente usadas para expressar investimentos não-tangíveis.

Resultados semelhantes são obtidos no estudo da Hipótese 3: RD e CAPEX estão positivamente relacionadas com a IDE, enquanto SGA não está relacionada com IDE. A hipótese 4 investiga a potencial relação entre retorno total ao acionista (TSR) e IDE, EARN e suas variações de um ano para outro, $\triangle$ IDE e $\triangle E A R N$. Os resultados mostram que todas as variáveis estão significativamente relacionadas com a TSR. No entanto, $\triangle \mathrm{IDE}$ e $\triangle E A R N$ têm influência negativa sobre o TSR, sugerindo que o valor para o acionista pode diminuir devido a uma variação positiva de IDE de um período para outro e para uma maior EBITDA no período anterior.

Tabela 2. Resultado dos modelos para hipóteses 1 a 4.

\begin{tabular}{|c|c|c|c|c|c|c|c|}
\hline Modelo & Hipótese 1 & Hipótese 2 & & Hipótese & & \multirow{2}{*}{\multicolumn{2}{|c|}{$\begin{array}{c}\text { Hipótese } 4 \\
\text { TCP }\end{array}$}} \\
\hline Variável Dependente & MV & IC & & IDE & & & \\
\hline Intercepto & -4780000 & 4680000 & *** & 80700 & *** & 0.182 & *** \\
\hline $\mathrm{CV}$ & 0.722 & & & & & & \\
\hline$R D$ & & 11.906 & * & 1.679 & $* * *$ & & \\
\hline CAPEX & & 4.706 & *** & 0.457 & *** & & \\
\hline SGA & & 0.255 & & -0.017 & & & \\
\hline IDE & & & & & & $1.61 \mathrm{E}-07$ & \\
\hline DIDE & & & & & & $-2.24 \mathrm{E}-07$ & *** \\
\hline EARN & & & & & & $-1.38 \mathrm{E}-07$ & *** \\
\hline GEARN & & & & & & $1.26 \mathrm{E}-07$ & * \\
\hline Especificação & Fixo & Fixo & & Fixo & & Aleatório & \\
\hline Estatística do Modelo & 14.96 & 18.22 & & 177.69 & & 11.10 & \\
\hline p-valor & 0.000 & 0.000 & & 0.000 & & 0.026 & \\
\hline $\mathrm{R}^{2}$ Within & 0.203 & 0.590 & & 0.455 & & 0.043 & \\
\hline$R^{2}$ Between & 0.715 & 0.878 & & 0.649 & & 0.040 & \\
\hline $\mathrm{R}^{2}$ Overall & 0.676 & 0.760 & & 0.588 & & 0.038 & \\
\hline
\end{tabular}

Fonte: elaboração dos autores. Obs: * significante ao nível de $10 \%$, ** significante ao nível de $5 \%$ e *** significante ao nível de $1 \%$.

Finalmente, as hipóteses de 5 a 9 analisam potenciais relações entre a criação de valor para os acionistas e os índices de intangibilidade propostos por Gu e Lev (2003). Neste contexto, a análise permite a investigação da existência ou não de algumas medidas intangibilidade para explicar retorno total ao acionista. Os resultados mostram que margem do capital intangível (ICM), a margem do capital intangível operacional (ICOM) e a razão entre o valor de mercado e o comprehensive value (MVR) são positivamente relacionados com o retorno total 
ao acionista. No entanto, a margem de ganhos intangíveis (IDEM), a razão entre - capital intangível e o valor contábil (ICR) não estão relacionados com o TSR. Portanto, apesar de IDE e IC potencialmente refletir os ativos intangíveis, o estudo da relação entre criação de valor e intangibilidade pode depender da escolha do índice de intangibilidade.

A hipótese 4 estabelece uma relação positiva entre IDE e TSR, sugerindo que quanto maior a intangibilidade maior a criação de valor para os acionistas, estudos futuros devem investigar como melhorar os índices de intangibilidade propostos por Gu e Lev (2003). É importante ressaltar como a amostra não é aleatória e, devido ao número limitado de observações, os resultados não podem ser generalizados. No entanto, o estudo apresenta evidências da importância dos intangíveis na criação de valor e uma análise empírica dos índices de intangibilidade definidos Gu e Lev (2003) em um setor específico do mercado americano.

Tabela 3. Resultado dos modelos para hipóteses 5 a 9.

\begin{tabular}{|c|c|c|c|c|c|}
\hline Modelo & Hipótese 5 & Hipótese 6 & Hipótese 7 & Hipótese 8 & Hipótese 9 \\
\hline $\begin{array}{c}\text { Variável } \\
\text { Dependente }\end{array}$ & TSR & TSR & TSR & TSR & TSR \\
\hline Intercepto & 0.357 & 0.329 & 0.302 & 0.348 & 0.309 \\
\hline$I C M$ & 0.011 & & & & \\
\hline IDEM & & 0.106 & & & \\
\hline ICOM & & & 0.044 & & \\
\hline ICR & & & & $\begin{array}{l}-1.53 \mathrm{E}- \\
04\end{array}$ & \\
\hline MVR & & & & & 0.068 \\
\hline Especificação & Fixo & Aleatório & Aleatório & Aleatório & Aleatório \\
\hline Estatística do & & & & & \\
\hline Modelo & 12.11 & 0.14 & 3.10 & 0.08 & 6.46 \\
\hline p-valor & 0.001 & 0.709 & 0.078 & 0.775 & 0.110 \\
\hline$R^{2}$ Within & 0.110 & 0.018 & 0.013 & 0.000 & 0.011 \\
\hline $\mathrm{R}^{2}$ Between & 0.029 & 0.003 & 0.053 & 0.008 & 0.164 \\
\hline $\mathrm{R}^{2}$ Overall & 0.001 & 0.000 & 0.027 & 0.001 & 0.062 \\
\hline
\end{tabular}

Fonte: elaboração dos autores. Obs: * significante ao nível de $10 \%$, ** significante ao nível de $5 \%$ e *** significante ao nível de $1 \%$.

\section{Considerações finais}

Os ativos intangíveis são cada vez mais importante no ambiente corporativo, tornando a sua mensuração crítica. Neste contexto, nossa pesquisa visou investigar componentes da intangibilidade através do modelo proposto por Gu e Lev (2003). Usando um banco de dados de empresas de consumidor de serviços dos Estados Unidos de 1998 a 2010, testamos nove hipóteses no modelo de dados em painel. 
Embora a amostra não seja aleatória e os resultados não podem ser generalizados, obtemos alguma evidência de uma relação positiva entre ativos intangíveis e a criação de valor. Nós achamos que o comprehensive value, ou seja, um indicador do valor total da empresa medida pelo capital intangível e valor contábil, está intimamente relacionado ao valor de mercado. Portanto, as construções de IDE e IC, associado com os ganhos gerados pelos intangíveis, pode refletir o valor atribuído pelos investidores para a empresa.

Nós também encontramos que os construtos de valor intangível propostos por Gu e Lev (2003), IDE e IC, estão positivamente relacionados às variáveis de demonstrações financeiras que são normalmente utilizados como proxies para intangíveis: pesquisa e desenvolvimento despesas e gastos de capital.

Em outras análises desta pesquisa, verificamos possíveis relações entre retorno total ao acionista e os índices formulados por Gu e Lev (2003). A criação de valor para os acionistas é positivamente relacionada com a ICM, ICOM e MVR, mas não para IDEM e ICR.

Estes resultados levantam questões importantes que devem ser estudados em pesquisas futuras: Os índices sugeridos por Gu e Lev (2003) de fato representam intangíveis? E se eles não representam intangíveis, por que alguns deles estão relacionados com a criação de valor e algumas não? Claro, essas questões não são fáceis de responder e, assim, o estudo de intangíveis ainda constitui um desafio para os pesquisadores, uma vez que a medição de algo não palpável pode ser difícil, mas muito necessário para compreender a criação de valor.

\section{Referências}

Daum JH. Intangible assets: Based enterprise management - a practical approach. PMA IC Symposium, 2005.

Domeneghetti D, Meir R. Ativos intangíveis: como sair do deserto competitivo dos mercados e encontrar um oásis de valor e resultados para sua empresa. Rio de Janeiro: Elsevier, 2009.

Edvinsson L, Malone MS. Intellectual capital: realizing your company's true value by finding its hidden brainpower. New York: Harper Business, 1997.

Gu F, Lev B. Intangible assets measurement, drivers, usefulness. Working Paper, Boston, 2003.

Lev B. Intangibles: management, measurement and reporting. Washington: Brooking Institution Press, 2001.

Low J, Kalafut PC. Intangible advantage: how intangibles are driving business performance. Cambridge: Perseus Books, 2002. 
Stewart TA. Intellectual capital: the new wealth of organizations. New York: Doubleday, 1997.

Sullivan PH. Value-driven intellectual capital: how to convert intangible corporate assets into market value. New York: John Wiley \& Sons, 2000.

Villalonga B. Intangible resources, Tobin's q, and sustainability of performance differences. J Econ Behavior \& Organiz. 2004, 54:205-230.

Young SD, O'Byrne SF. EVA and value-based management: a pratical guide to implementation. New York: McGraw-Hill, 2001. 